\title{
Patient safety incident capture resulting from incident reports: a comparative observational analysis
}

Martin A Reznek, Kevin A Kotkowski, Michael W Arce, Zachary K Jepson, Steven B Bird and Chad E Darling

\begin{abstract}
Background: Patient safety incident (PSI) discovery is an essential component of quality improvement. When submitted, incident reports may provide valuable opportunities for PSI discovery. However, little objective information is available to date to quantify or demonstrate this value. The objective of this investigation was to assess how often Emergency Department (ED) incident reports submitted by different sources led to the discovery of PSIs.
\end{abstract}

Methods: A standardized peer review process was implemented to evaluate all incident reports submitted to the ED. Findings of the peer review analysis were recorded prospectively in a quality improvement database. A retrospective analysis of the quality improvement database was performed to calculate the PSI capture rates for incident reports submitted by different source groups.

Results: 363 incident reports were analyzed over a period of 18 months; 211 were submitted by healthcare providers (HCPs) and 126 by non-HCPs. PSIs were identified in 108 resulting in an overall capture rate of $31 \%$. HCP-generated reports resulted in a $44 \%$ capture rate compared to $10 \%$ for non-HCPs $(p<0.001)$. There was no difference in PSI capture between sub-groups of HCPs and non-HCPs.

Conclusion: HCP-generated ED incident reports were much more likely to capture PSIs than reports submitted by non-HCPs. However, HCP reports still led to PSI discovery less than half the time. Further research is warranted to develop effective strategies to improve the utility of incident reports from both HCPs and non-HCPs.

Keywords: Incident reporting, Quality improvement, Patient safety, Emergency medicine

\section{Background}

In 1999, the Institute of Medicine presented startling findings that 44,000 to 98,000 patients die annually due to medical errors [1]. Since that report, patient safety has become a major priority in healthcare.

Incident reporting is important for improving patient safety because it can lead to the identification of patient safety incidents (PSIs), defined by the World Health Organization as "event[s] or circumstance[s] that could have resulted, or did result, in unnecessary harm to a patient" [2]. PSI capture is a crucial step in patient safety improvement because it enables subsequent development and implementation of preventative measures [3].

\footnotetext{
* Correspondence: mreznek@hotmail.com

Department of Emergency Medicine, University of Massachusetts Medical School, 55 Lake Avenue North, Worcester, MA 01655, USA
}

In Emergency Medicine (EM), potential incidents are reported to Emergency Department (ED) leadership from a wide variety of sources including ED healthcare providers (HCPs), HCPs in other departments, hospital quality officers, hospital committees, as well as patients and their families. These reports may represent valuable opportunities for discovery of true PSIs, however little objective information is available to quantify or demonstrate this value. In EM, a limited number of studies suggest that patient complaints may be useful in improving quality $[4,5]$, and one study has implied that physiciangenerated concerns may be more valuable for quality improvement [6]. To our knowledge however, there are no studies in any field of healthcare that have specifically evaluated the efficacy of incident reports from different sources in identifying PSIs. 
The objective of this investigation was to assess how often ED incident reports from different sources led to the identification of PSIs.

\section{Methods}

\section{Study design and setting}

Data for this observational study were collected between October 2010 and April 2012 at an urban, tertiary care ED with an approximate annual census of 66,000 adult patients. The ED is the primary teaching site for an accredited 3-year EM residency. Board-certified EM physicians care directly for approximately $95 \%$ of the patients either as the only provider or through direct supervision of EM and rotating residents. Mid-level practitioners care for approximately $5 \%$ of the patients as the primary provider. The University of Massachusetts Medical School Institutional Review Board reviewed and approved the study.

\section{Methods and measurements}

To analyze incident reports submitted to the ED, a standardized peer review process was implemented in October 2010. The process was based on one previously described at Detroit Receiving Hospital (DRH) [7], however we modified the DRH process also to include systems failure identification and analysis. As a matter of policy, every incident report submitted to the ED was analyzed via this process which is described below.

As a first step, each incident report underwent a preliminary screening review. Due to practical considerations, we opted for a single-reviewer format for the preliminary screening. To minimize potential accuracy and bias limitations inherent to a single-reviewer screening, the chosen preliminary reviewer had greater than ten years of experience in healthcare quality and the incident reports could only be closed if it could be concluded with absolute certainty that no PSI had occurred. If a PSI could not be excluded with absolute certainty, the incident report was forwarded to the ED Peer Review Committee (PRC) for analysis.

Convening monthly, the PRC was open to all ED physicians, residents and mid-level practitioners, however eight board-certified, attending physicians attended regularly to provide consistency in the evaluation process. Prior to each PRC meeting, ED practitioners involved with a case to be reviewed were required to provide a written account of the care and any other circumstances related to the specific quality issue(s) in question. This allowed for first-hand accounts of the care of the patient that might not be readily available in the medical record to be available to the PRC. In addition, the involved providers were encouraged to attend the PRC meeting. The medical records (and the practitioners' written feedback if they were not present) were de-identified to foster a non-punitive and objective environment and to maintain patient confidentiality.

Facilitated by the PRC Chair, the committee reviewed each case for potential PSIs which were further subdivided by the PRC into six types of systems failures or contributing factors and five types of practitioner-based errors (Table 1). The classification scheme was developed based on schemes previously reported in EM literature. The classification framework of practitionerbased errors was modeled on one reported by Berk et al. [7]. For systems failures, the framework was developed based on portions of one suggested by Cosby [8]. Within the PRC review process, determination of a failure or error occurred regardless of correlation or causation of patient harm, and therefore near-misses were included as PSIs. A majority vote determined the presence or absence of systems failures and/or practitioner-based errors. (Providers involved in a case were required to leave the room during the vote on practitioner-based error for that case in order to reduce possible influence on the objectivity of the process.) To clarify the PSI identification and classification process, the following scenario is used as an example. A patient is admitted from the ED to an in-patient unit, and the patient's condition subsequently deteriorates shortly after admission. The admitting team submits a concern that the care in the ED may have been suboptimal. Upon investigation, the ED peer review committee may determine that a systems failure did occur such as the patient did not receive an ordered medication due to a communication error. Or it may be determined after review, that the care was optimal, no systems failures or practitioner-based errors occurred, and the patient had simply deteriorated due to the natural course of disease. In the former outcome, a PSI was determined to have occurred, and in the latter outcome, it was determined that there was no PSI.

All incident reports received by the ED during the study period were recorded prospectively in a secure, quality improvement database. The data included the respective source of each incident report and the results of the peer review analysis. The entire peer review process, including the database and practitioner written responses, was structured so that it complied with state peer review legal discoverability protection requirements.

Sources of incident reports were assigned to one of two broad source group categories: those that were generated by health care providers (HCPs) and those that were from non-health care providers (non-HCPs). The HCP group was sub-divided into four groups: Self (ED provider self-referral), ED (ED provider), HP (Hospital provider) and $\mathrm{OP}$ (Other provider outside the hospital), and the non-HCP group was subdivided into three groups: PFM (Patient and family members), Admin (Hospital administration), and Risk (Risk management) for a total of 
Table 1 Patient safety incident types identified by the peer review committee

\begin{tabular}{|c|c|c|}
\hline PSI type & Definition & Example \\
\hline \multicolumn{3}{|l|}{ Systems failures } \\
\hline Triage & A failure in assessment of potential disease severity during triage & $\begin{array}{l}\text { Abnormal vital signs not recognized as a } \\
\text { potential sign of shock }\end{array}$ \\
\hline ED teamwork & $\begin{array}{l}\text { A failure due to an issue with ED staff communication or a shared responsibility } \\
\text { across multiple ED staff }\end{array}$ & $\begin{array}{l}\text { Change in vital signs not communicated } \\
\text { to the attending physician }\end{array}$ \\
\hline $\begin{array}{l}\text { Hospital } \\
\text { Teamwork }\end{array}$ & $\begin{array}{l}\text { A failure due to an issue with communication between ED and hospital } \\
\text { staff or a shared responsibility between the ED and hospital staff }\end{array}$ & $\begin{array}{l}\text { Pertinent information not communicated } \\
\text { to the admitting team }\end{array}$ \\
\hline ED work environment & $\begin{array}{l}\text { A failure resulting from the lack, malfunction, or mal-design of resources, } \\
\text { equipment or physical space within the ED or a failure resulting from } \\
\text { following an established ED policy or clinical practice guideline }\end{array}$ & $\begin{array}{l}\text { Missing equipment needed for care of } \\
\text { a patient }\end{array}$ \\
\hline $\begin{array}{l}\text { Hospital work } \\
\text { environment }\end{array}$ & $\begin{array}{l}\text { A failure resulting from the lack, malfunction, or mal-design of resources } \\
\text { equipment or physical plant outside the ED but still within the hospital or } \\
\text { a failure resulting from following an established hospital policy or guideline }\end{array}$ & $\begin{array}{l}\text { Specialty testing areas remotely located } \\
\text { from the ED causing prolonged } \\
\text { transport time }\end{array}$ \\
\hline Boarded patient & $\begin{array}{l}\text { A failure occurred after the patient was admitted to an in-patient service but } \\
\text { was still physically in the ED }\end{array}$ & N/A \\
\hline \multicolumn{3}{|c|}{ Practitioner-based errors } \\
\hline Major cognitive error & $\begin{array}{l}\text { An error in cognition which represents serious mismanagement in a knowledge } \\
\text { area basic to emergency }\end{array}$ & $\begin{array}{l}\text { Failure to diagnose and treat ST-elevation } \\
\text { myocardial infarction }\end{array}$ \\
\hline Cognitive error & $\begin{array}{l}\text { An error in cognition which represents mismanagement which is either less } \\
\text { serious than a major cognitive error or in an area less basic to emergency } \\
\text { medicine }\end{array}$ & $\begin{array}{l}\text { Failure to consider the institutional } \\
\text { antibiogram during antibiotic selection } \\
\text { for treatment }\end{array}$ \\
\hline $\begin{array}{l}\text { Missed radiographic } \\
\text { finding }\end{array}$ & $\begin{array}{l}\text { An error in interpretation of a radiographic study that did not reach the level } \\
\text { of a cognitive or major cognitive error }\end{array}$ & $\begin{array}{l}\text { Missed fracture on radiographic } \\
\text { interpretation that was still splinted } \\
\text { based on clinical suspicion }\end{array}$ \\
\hline Policy deviation & $\begin{array}{l}\text { An error in following a clinical or administrative policy, guideline or standard } \\
\text { practice that does not reach the level of cognitive or major cognitive error }\end{array}$ & $\begin{array}{l}\text { Failure to alert the transplant service } \\
\text { when a transplant patient is in the ED }\end{array}$ \\
\hline Procedural error & $\begin{array}{l}\text { A technical error during performance of a procedure that does not reach the } \\
\text { level of a cognitive or major cognitive error }\end{array}$ & Insufficient sterile technique \\
\hline
\end{tabular}

seven sub-groups (Table 2). In the case that reports were submitted by multiple sources for one case, the first report received by ED leadership was identified as the source.

For the present study, a retrospective analysis of the existing peer review database was performed to determine the PSI capture rate of incident reports from each of the groups and sub-groups. Specifically, each incident report in the database was reviewed to determine the source group and sub-group as well as if any failures or errors were identified for that case indicating that a PSI had occurred.

\section{Outcomes and analysis}

The primary outcome of this study was the frequency of PSI capture based on the source of the incident report. Frequency of PSI capture was expressed as a percent. A two-tailed Fisher's exact test was used to determine if the capture rates differed between the HCP and nonHCP groups. We similarly compared the frequency of PSI capture within the sub-groups.

\section{Results}

Over a period of 18 months, 353 incident reports were submitted to the ED, and 108 (31\%) resulted in the

\section{Table 2 Sources of incident reporting}

\begin{tabular}{ll}
\hline Source & Definition \\
\hline Self & An ED practitioner who was directly involved in the care of the patient when the perceived patient safety incident occurred \\
ED & An ED practitioner not directly involved in the care of the patient at the time that the perceived patient safety incident occurred \\
HP & A practitioner within the hospital that is not an ED practitioner or a clinical committee within the hospital (for example - the Stroke \\
OP & Care Committee) \\
PFM & A patient or family member \\
Admin & Central hospital management forwarding a concern from an outside agency (for example - an insurance company generated concern) \\
Risk & Risk management; a hospital administrative unit responsible for risk assessment and quality management. \\
\hline
\end{tabular}


discovery of one or more PSIs. Of the 353 reports, 351 were classified into a source group (Table 2). The reporting source was not recorded in two cases in the database, so they were classified as "unknown". The frequency of reports for each source group is summarized in Table 3. HCPs submitted 211 reports of which 94 led to PSI capture (44.5\%), and non-HCPs submitted 126 reports of which 14 led to PSI capture (10.0\%). HCP-generated reports resulted in PSI capture more frequently than those generated by non-HCPs $(\mathrm{p}<0.0001)$.

Among the subcategories of HCPs and non-HCPs, PSI capture ranged from $41.7-45.0 \%$ and $0-20.0 \%$ respectively. Reports submitted by the four different HCP subcategories and by the three different non-HCP subcategories did not demonstrate within-group differences with respect to the frequency of PSI capture ( $\mathrm{p}=0.99$ and $\mathrm{p}=0.14$ respectively) (Table 3).

\section{Discussion}

The results of this investigation of ED incident reports revealed two key findings. First, incident reports submitted by HCPs led to the capture of PSIs nearly 4.5 times more frequently than those submitted by non-HCPs. And second, while $\mathrm{HCP}$-generated incident reports were more likely to result in PSI capture, they resulted in capture only less than half the time.

Given that HCPs are likely to have more insight into healthcare delivery systems than non-HCPs, the finding that HCP-generated reports led more frequently to PSI discovery may not be altogether surprising. More remarkable however was the finding of over a four-fold difference in resultant PSI discovery between these groups. The non-HCP category in our sample was dominated by reports submitted by patients and families. Data from previous investigations have suggested that patient and family concerns often are related to incomplete or delayed relief in symptoms, suboptimal practitioner communication or billing related to their medical services $[4,5,9]$. The design of this study did not address this topic directly as the specific concerns prompting the incident reports were not systematically recorded in the database, however our experience was consistent with these previous investigations. Of note, while concerns generated by patients and families most often did not lead to PSI identification, we feel strongly that their feedback is still highly valuable for understanding and improving the patient experience.

With regards to this investigation's second key finding of HCP-generated incident reports capturing PSIs 44.5\% of the time, the reasons for this seemingly low capture rate are unclear. Reports from each HCP sub-group resulted in PSI identification at a similar frequency regardless of the HCP's role or if they were directly involved in the care in question. For providers not directly involved in the care in question (ED, HP and OP), the relatively low percentage of capture could be due to the fact that they were not present during the care in question and therefore were biased toward presuming that an adverse outcome was due to a preventable failure or error. However, this does not explain the capture rate for selfreporting. Perhaps self-reporters were biased by emotion or a sense of personal responsibility when adverse outcomes occurred. In any case, the observed overreporting by all types of HCPs can be interpreted as a promising sign of their engagement in reducing PSIs. The observed over-reporting by HCPs in this study may appear to contradict previous studies suggesting that HCP under-reporting is a common barrier to improving patient safety $[3,9]$, however the two phenomena are not necessarily mutually exclusive. Based on the prior literature and the results of this study, it is likely that HCP under-reporting and over-reporting are occurring simultaneously. The implication is that HCPs are engaged in patient safety improvement, but there is opportunity to better educate them about patient safety. Optimizing

Table 3 Incident reports and patient safety incident capture by source group

\begin{tabular}{|c|c|c|c|c|c|}
\hline Source group & Number of reports & Percent of total reports & Number resulting in PSI identification & PSI capture rate & $\mathrm{p}$-value \\
\hline \multicolumn{6}{|c|}{ Healthcare Providers (HCP) $n=211$} \\
\hline Self & 40 & $11.3 \%$ & 18 & $45.0 \%(18 / 40)$ & \multirow[t]{4}{*}{$p=0.99$} \\
\hline ED & 69 & $19.5 \%$ & 31 & $44.9 \%(31 / 69)$ & \\
\hline $\mathrm{HP}$ & 90 & $25.5 \%$ & 40 & $44.4 \%(40 / 90)$ & \\
\hline $\mathrm{OP}$ & 12 & $3.4 \%$ & 5 & $41.7 \%(5 / 12)$ & \\
\hline \multicolumn{6}{|c|}{ Non-Healthcare Providers (Non-HCP) $n=140$} \\
\hline PFM & 112 & $31.7 \%$ & 9 & $8.0 \%(9 / 112)$ & \multirow[t]{3}{*}{$p=0.14$} \\
\hline Admin & 4 & $1.1 \%$ & 0 & $0.0 \%(0 / 0)$ & \\
\hline Risk & 24 & $6.8 \%$ & 5 & $20.8 \%(5 / 24)$ & \\
\hline \multicolumn{6}{|l|}{ Other } \\
\hline Unknown & 2 & $0.6 \%$ & 0 & $0.0 \%(0 / 2)$ & $\mathrm{n} / \mathrm{a}$ \\
\hline Overall Total & 353 & $100 \%$ & 108 & $30.6 \%(108 / 353)$ & $\mathrm{n} / \mathrm{a}$ \\
\hline
\end{tabular}


practitioner education in this area may improve over and under-reporting simultaneously. Ultimately reaching a capture rate of $100 \%$ may be unrealistic. Furthermore, if a perfect capture rate is attained, it is likely that incidents are going under-reported. To our knowledge, this is the first study reporting capture rates from incident reports, so there are no other data to assist with benchmarking. While we cannot definitively conclude that the rate observed in this study is low, we believe that a capture rate of $44.5 \%$ from HCP incident reports can be improved. It stands to reason that the ideal rate may be between the observed rate and $100 \%$. In our institution, we are considering an initial goal of $75 \%$ as we continue to improve our incident reporting processes.

While education related to patient safety may be a viable mechanism to improve the PSI capture rate for HCP incident reports, the mechanism to improve them for non-HCP reports is less clear. A prior study reported utilizing structured patient interviews to elicit ED patient feedback rather than self-generated concerns, however the capture rate was still low (9\%) [10]. Despite the low rate similar to that observed in our study, we believe that structured patient interviews may still be a promising option. To improve the capture rate of patient and family incident reporting, perhaps the structured interview tool previously reported requires redesign or perhaps follow-up interviews should be directed only toward patients that are high risk for having been subject to a PSI.

Incident reporting by HCPs as well as patients and family members traditionally has been cited as an important tool for PSI identification [9,11-14]. Recently however, some research has shown that while incident reporting can detect latent errors, it may miss a large fraction of PSIs [15]. More proactive and systematic surveillance techniques have been suggested as superior for improving patient safety [15-18] Systematic surveillance techniques however may have potential shortcomings including the fact that they may miss PSIs that do not fit strict systematic surveillance criteria $[15,16]$. For this reason, we believe that incident reporting and systematic surveillance complement each other, and there is in fact a role for both in healthcare quality improvement [19]. Therefore, gaining a better understanding of incident reporting is important so that we may continue to improve the effectiveness of this incident identification modality.

Previously we have published results of an investigation of the type, frequency, and resultant harm related to PSIs that were discovered via the ED-based incident reporting system [20]. The purpose of the present study was not to review these details again but to investigate the utility of incident reports from different sources in leading to PSI discovery in general. Nevertheless, it is worth noting that in the prior publication, we sought to broadly classify PSIs into those related to either practitioner-based error or systems failures. We are aware that this approach differs from the more detailed categorization scheme used by the National Health Service in the United Kingdom well as that which has been proposed by the World Health Organization [21,22]. Therefore, directly comparing our findings of PSI types with either of those systems would be difficult.

This study of incident reports has potential limitations that warrant consideration when interpreting the results. First, the peer review process was designed to be systematic and as objective as possible; however any review process of incident reports necessarily must involve human interpretation. Barriers for impartial decision making among peers are known to exist, and hindsight bias is likely unavoidable in determining medical errors [23]. A range of measures were designed into the review process to mitigate the influence of biases (discussed in the Methods section), but having completely removed all bias was unlikely. A second potential limitation of the study was the fact that the peer review database only included the original source when more than one report for a case was received. In our anecdotal experience however, very few cases involved reports from both HCPs and non-HCPs, and there was no apparent trend in whether HCPs or non-HCPs were the first to report. The reason for this may have been that non-HCPs also had easily accessible mechanisms to submit incident reports in our institution. In addition to other mechanisms, non-HCPs could submit concerns verbally to the "Patient Care Services" department at any time including at the time of service. Another potential limitation of this study was that it was performed at a single center and focused on EM as opposed to multiple medical specialties. With regard to the generalizability of our study within EM, the frequency of incident reports we observed $(0.36 \%$ of ED patient encounters) is consistent with other published ED studies which cite a range of $0.012 \%$ to $0.61 \%$ in EDs with 13,000 to 94,000 patient encounters per year $[24,25]$. While it cannot be determined if the distribution of incident reporter type is consistent with other EDs, the overall frequency of incident reporting is similar to other reports in the literature. The applicability of this study beyond EM is less clear. It may be the case that incident reporting is more likely to occur in EDs because nearly all care is handed off to other HCPs who naturally review the care that has been delivered prior to their involvement. However the fact that incident reports from non-ED HCPs resulted in similar PSI capture rates as those from ED HCPs in the study provides some promising evidence that the results may be generalizable to other specialties. 


\section{Conclusion}

Any incident report, regardless of its source, has the potential to lead to PSI discovery and ultimately result in quality improvement. This study revealed that HCPgenerated reports led to PSI capture much more frequently than reports from other sources; however they still resulted in capture less than half the time. These two observations together provide promising evidence that there may be opportunity to further improve the already valuable feedback from HCPs and that quality improvement programs may likely benefit by prioritizing strategies to further increase and optimize feedback from this group. By comparison, non-HCP generated concerns appear to be relatively less valuable for PSI identification. Quality improvement leaders might consider implementing programs to solicit directed feedback from patients and families in order to improve the patient safety incident capture rate, however the potential benefit of these efforts remains unclear to date. Further research is warranted to:

1) determine the most effective strategies to further increase and improve HCP incident reporting

2) determine if soliciting directed patient and family feedback may improve PSI capture and

3) determine if patient feedback systems targeted at subgroups of ED patients that are at higher risk of safety incidents may improve PSI capture rates from this group.

\section{Competing interests}

The authors declare that they have no competing interests.

\begin{abstract}
Authors' contributions
MAR developed the study concept and design, acquired, analysed and interpreted the data, drafted the manuscript and supervised the study; KAK analysed and interpreted the data and critically revised the manuscript for important intellectual content; MWA acquired the data and assisted with drafting the manuscript; ZKJ acquired the data and assisted with drafting the manuscript; SBB performed statistical analysis of the data and critically revised the manuscript for important intellectual content; CED analysed and interpreted the data, drafted the manuscript and supervised the study. All authors participated in the peer review committee. All authors read and approved the final manuscript.
\end{abstract}

\begin{abstract}
Authors' information
MAR was the Vice-Chairman for Clinical Affairs in the Department of EM. He has also served previously as a Vice President of Quality and Patient Safety in a large, urban, teaching hospital. KAK was the Fellow in EM Administration and Leadership. MWA and ZKJ were residents in the Department with interests in EM administration. SBB was Vice-Chairman of Academic Affairs and the Residency Program Director. CED was an Associate Professor of EM with diverse research experience in various EM topics and an interest in quality improvement.
\end{abstract}

Received: 19 March 2014 Accepted: 24 March 2015

Published online: 11 April 2015

\section{References}

1. Kohn LT, Corrigan JM, Donaldson MS, editors. To Err Is Human: Building a Safer Health System. Washington, DC: National Academy Press, Institute of Medicine; 1999.
2. WHO. More than words: Conceptual framework for the international classification for patient safety; final technical report; 2009. Available at: http://www.who.int/patientsafety/taxonomy/icps_full_report.pdf.

3. Leape LL. Errors in medicine. Clin Chim Acta. 2009;404:2-5.

4. Chande VT, Bhende MS, Davis HW. Pediatric emergency department complaints: a three-year analysis of sources and trends. Ann Emerg Med. 1991;20:1014-6.

5. Schwartz LR, Overton DT. Emergency department complaints: a one-year analysis. Ann Emerg Med. 1987;16:857-61.

6. Griffey RT, Bohan JS. Healthcare provider complaints to the emergency department: a preliminary report on a new quality improvement instrument. Qual Saf Health Care. 2006;15:344-6.

7. Berk WA, Welch RD, Levy PD, Jones JT, Arthur C, Kuhn GJ, et al. The effect of clinical experience on the error rate of emergency physicians. Ann Emerg Med. 2008;52:497-501.

8. Cosby KS. A framework for classifying factors that contribute to error in the emergency department. Ann Emerg Med. 2003:42:815-23.

9. Schenkel S. Promoting patient safety and preventing medical error in emergency departments. Acad Emerg Med. 2000;7:1204-22.

10. Friedman SM, Provan D, Moore S, Hanneman K. Errors, near misses and adverse events in the emergency department: what can patients tell us? CJEM. 2008;10:421-7.

11. Department of Health. An Organisation with a Memory: Report of an Expert Group on Learning from Adverse Events in the NHS. London: The Stationery Office; 2000 .

12. Petersen LA, Orav EJ, Teich JM, O'Neil AC, Brennan TA. Using a computerized sign-out program to improve continuity of inpatient care and prevent adverse events. Jt Comm J Qual Improv. 1998;24:77-87.

13. Weingart SN, Pagovich O, Sands DZ, Li JM, Aronson MD, Davis RB, et al. What can hospitalized patients tell us about adverse events? Learning from patient-reported incidents. J Gen Intern Med. 2005;20:830-6.

14. Weingart SN, Zhu J, Chiappetta L, Stuver S, Schneider E, Epstein A, et al. Hospitalized patients' participation and its impact on quality of care and patient safety. Int J Qual Health Care. 2011;23:269-77.

15. Vincent C. Incident reporting and patient safety. BMJ. 2007;334:51.

16. Thomas EJ, Petersen LA. Measuring errors and adverse events in health care. J Gen Intern Med. 2003;18:61-7.

17. Sari AB, Sheldon TA, Cracknell A, Turnbull A. Sensitivity of routine system for reporting patient safety incidents in an NHS hospital: retrospective patient case note review. BMJ. 2007;334:79.

18. Hospital incident reporting systems do not capture most patient harm: OIG, Department of Health and Human Services. 2012. Available at: http://oig.hhs.gov/oei/reports/oei-06-09-00091.asp.

19. Olsen S, Neale G, Schwab K, Psaila B, Patel T, Chapman EJ, et al. Hospital staff should use more than one method to detect adverse events and potential adverse events: incident reporting, pharmacist surveillance and local real-time record review may all have a place. Qual Saf Health Care. 2007;16:40-4.

20. Jepson ZK, Darling CE, Kotkowski KA, Bird SB, Arce MW, Volturo GA, et al. Emergency department patient safety incident characterization: an observational analysis of the findings of a standardized peer review process. BMC Emerg Med. 2014;14:20.

21. World Health Organization. Conceptual framework for the international classification of patient safety. Version 1.1. 2009. Retrieved from http://www. who.int/patientsafety/taxonomy/icps_full_report.pdf.

22. NHS. National Reporting and Learning System (NRLS). http://www.nrls.npsa. nhs.uk/. Accessed January 7, 2015

23. Wears RL, Nemeth CP. Replacing hindsight with insight: toward better understanding of diagnostic failures. Ann Emerg Med. 2007:49:206-9.

24. Chamberlain JM, Shaw KN, Lillis KA, Mahajan PV, Ruddy RM, Lichenstein R, et al. Creating an infrastructure for safety event reporting and analysis in a multicenter pediatric emergency department network. Pediatr Emerg Care. 2013;29:125-30

25. Considine J, Mitchell B, Stergiou HE. Frequency and nature of reported incidents during Emergency Department care. Emerg Med J. 2011;28:416-21. 